\title{
CORRELATIONS BETWEEN ELECTRONIC AND MAGNETIC PROPERTIES IN SEMIMAGNETIC SEMICONDUCTORS
}

\author{
T. STORY \\ Institute of Physics, Polish Academy of Sciences \\ Al. Lotników 32/46, 02-668 Warsaw, Poland
}

\begin{abstract}
Magnetic properties of IV-VI semimagnetic (diluted magnetic) semiconductors with $\mathrm{Mn}$ or with $\mathrm{Gd}$ can be controlled by changing the electronic parameters such as carrier concentration, Fermi level position or density of states at the Fermi level. The examples of these effects are the carrier concentration induced paramagnet-ferromagnet and ferromagnet-spin glass transitions in PbSnMnTe and the Fermi level position controlled $\mathrm{Gd}-\mathrm{Gd}$ exchange interactions in SnGdTe.
\end{abstract}

PACS numbers: $75.20 . \mathrm{Ck}, 75.30 . \mathrm{t} t$

\section{Introduction}

Semimagnetic (diluted magnetic) semiconductors are substitutional solid solutions of the well-known II-VI, IV-VI and III-V semiconductors and magnetic semiconductors like, e.g., MnTe or GdTe [1-4]. Apart from the technological definition given above, the concept of semimagnetic semiconductor involves the formation (by magnetic ions) of the well-localized magnetic moments and the existence of the $s p-d(s p-f) J_{s d} S \sigma$ exchange coupling between these magnetic moments $S$ and the spin of conduction or valence band electrons $\sigma$. The most interesting effects related to the influence of electronic properties of semimagnetic semiconductors on their magnetic behaviour are observed in IV-VI group of semiconductors like $\mathrm{Sn}_{1-x} \mathrm{Gd}_{x} \mathrm{Te}$ or $\mathrm{Sn}_{1-x} \mathrm{Mn}_{x} \mathrm{Te}$. The review of the magnetic properties of these crystals is the primary subject of this paper.

The IV-VI group of semimagnetic semiconductors consists of the (best studied) Mn-based systems as well as the $\mathrm{Gd}, \mathrm{Eu}, \mathrm{Yb}, \mathrm{U}$, and Cr-based systems. The spectrum of IV-VI matrices covers the $\mathrm{Pb}, \mathrm{Sn}$, and Ge tellurides, selenides and sulfides (like $\mathrm{PbSe}$ or $\mathrm{SnTe}$ ) as well as their alloys (like $\mathrm{Pb}_{1-y} \mathrm{Sn}_{y} \mathrm{Te}$ ). The solubility limit for these magnetic ions is typically about 10 at.\% with the exception of $\mathrm{Cr}$ where this limit is below 1 at.\% [1,5-8]. Most of the IV-VI semimagnetic semiconductors have the rock-salt crystal structure with lattice constants in the range $a_{0}=6.0 \div 6.5 \AA$. 
The electronic properties of IV-VI semimagnetic semiconductors are qualitatively similar to the properties of the host IV-VI crystals. These materials are strongly degenerated narrow-gap semiconductors with the energy gaps $E_{\mathrm{g}}=$ $0 \div 0.5 \mathrm{eV}$, depending on the chemical composition. They are known to have semimetallic electric properties. Carriers are generated by electrically active native defects (vacancies). Their concentration is temperature independent and can be controlled (by annealing) within 1-2 orders of magnitude [9]. The important role in both magnetic and electronic properties of IV-VI semiconductors is played by the band of heavy holes. The top of this band is located about $0.2 \mathrm{eV}$ below the top of the light hole band (see Fig. 1a). The density of states effective mass of heavy holes is an order of magnitude larger than the effective mass of light holes.
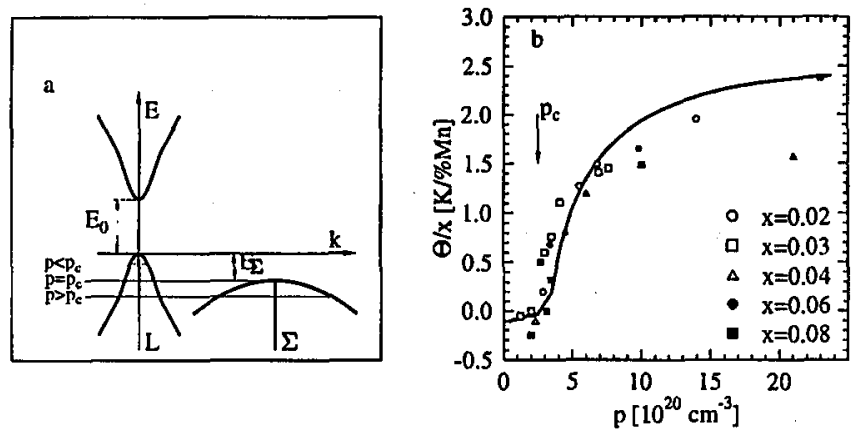

Fig. 1. (a) A model of the band structure of PbSnMnTe. The band of heavy holes (at $\Sigma$-point of the Brillouin zone) starts to be populated for carrier concentration $p=p_{c}$, where $E_{\mathrm{F}}\left(p_{\mathrm{c}}\right)=E_{\Sigma}$. (b) The carrier concentration dependence of the normalized Curie-Weiss temperature of $\mathrm{Pb}_{1-x-y} \mathrm{Sn}_{y} \mathrm{Mn}_{x} \mathrm{Te}(y=0.72,0.02 \leq x \leq 0.08)$. The solid line is the result of theoretical calculations based on the RKKY interaction due to both the light ( $L$-band) and the heavy ( $\Sigma$-band) holes.

\section{Mn-based IV-VI semimagnetic semiconductors}

The experimental investigations of transport, optical and magnetic properties as well as the analysis of electron paramagnetic resonance spectra of IV-VI semiconductors with $\mathrm{Mn}$ provide clear evidence that $\mathrm{Mn}$ incorporates into the IV-VI matrices as electrically neutral but magnetically active $\mathrm{Mn}^{2+}$ ion, possessing a local spin-only magnetic moment with $S=5 / 2$ [1-14]. The investigations of magnetic and magnetooptical properties of $\mathrm{Mn}$-based IV-VI semimagnetic semiconductors provided a solid evidence for the existence of the $s p-d$ exchange interaction between the system of local magnetic moments of $\mathrm{Mn}$ ions and the system of free carriers with the exchange integral $\mathrm{J}_{s d}$ of the order of $50-100 \mathrm{meV}[6-7,14-15]$.

\subsection{Carrier concentration induced magnetic phase transitions}

The magnetic properties of Mn-based IV-VI semimagnetic semiconductors have been studied using measurements of magnetization, ac magnetic susceptibility, specific heat, electron paramagnetic resonance, ferromagnetic resonance, 
anomalous Hall effect and neutron diffraction, and have been found to depend on both Mn concentration and carrier concentration.

The experimental studies of the magnetic properties of $\mathrm{Pb}_{1-x} \mathrm{Mn}_{x} \mathrm{Te}$ and related crystals with typical carrier concentrations $n, p=10^{17} \div 10^{19} \mathrm{~cm}^{-3}$ revealed that these materials are Curie-Weiss paramagnets down to $T=1 \mathrm{~K}$. The presence of a weak antiferromagnetic $d-d$ exchange interactions between $\mathrm{Mn}$ ions was observed both in the temperature dependence of magnetic susceptibility and in the magnetic field dependence of magnetization [5,16-17]. No carrier concentration dependence of magnetic properties was observed in these crystals. The weak antiferromagnetic exchange interactions were attributed to the superexchange via anions, the mechanism well known in, e.g., II-VI semimagnetic semiconductors.

The magnetic properties of crystals of $\mathrm{Sn}_{1-x} \mathrm{Mn}_{x} \mathrm{Te}$ and $\mathrm{Ge}_{1-x} \mathrm{Mn}_{x} \mathrm{Te}$ with typical carrier concentrations of the order of $p=10^{21} \mathrm{~cm}^{-3}$ are very different. These crystals are ferromagnetic at low temperatures. The decisive role of the free carriers in the formation of ferromagnetic phase was explicitly demonstrated for crystals of $\mathrm{Pb}_{1-x-y} \mathrm{Sn}_{y} \mathrm{Mn}_{x}$ Te for which the annealing allows one to control the concentration of carriers in the range $p=5 \times 10^{19} \div 2 \times 10^{21} \mathrm{~cm}^{-3}$, giving a possibility to study the evolution of magnetic properties as a function of the carrier concentration [18-21]. The ferromagnetic properties of the high carrier concentration IV-VI semimagnetic semiconductors are related to the Ruderman-Kittel-KasuyaYoshida (RKKY) indirect exchange interaction via free carriers [2,18-19,22-23]. The argument in favour of this mechanism is its explicit dependence on carrier concentration and its long-range character allowing for the creation of the ferromagnetic order even in quite diluted magnetic systems with only 1 at.\% of magnetic ions. The threshold-like dependence of Curie temperature on carrier concentration (referred as carrier concentration induced paramagnet-ferromagnet transition) can be explained within the framework of the RKKY exchange interaction and the band structure model of PbSnMnTe presented in Fig. 1a. The threshold carrier concentration $p_{\mathrm{c}}$ corresponds to the concentration of carriers for which the Fermi level enters the band of heavy holes (see Fig. 1) and these carriers start contributing to the RKKY mechanism. Since the effective mass of heavy holes is about 20 times larger than the effective mass of light holes the RKKY interaction is strongly enhanced being able to create the ferromagnetic order at low temperatures $[19,22]$.

With the further increase in the concentration of carriers (above $p=$ $10^{21} \mathrm{~cm}^{-3}$ ) the other carrier concentration induced the effect is experimentally observed. With the increasing carrier concentration one observes the breakdown of long-range ferromagnetic order and a gradual formation of a spin-glass state [24]. This process is clearly evidenced in the temperature dependence of magnetization, ac magnetic susceptibility and magnetic contribution to the specific heat (see Fig. 2) as well as in neutron diffraction studies [20]. This effect is also a consequence of the properties of the RKKY interaction as will be discussed in the next section.

\section{$2.2(x-p)$ magnetic phase diagram}

The experimental data on magnetic properties of Mn-based semimagnetic semiconductors can be summarized in the form of $x-p$ magnetic phase diagram 


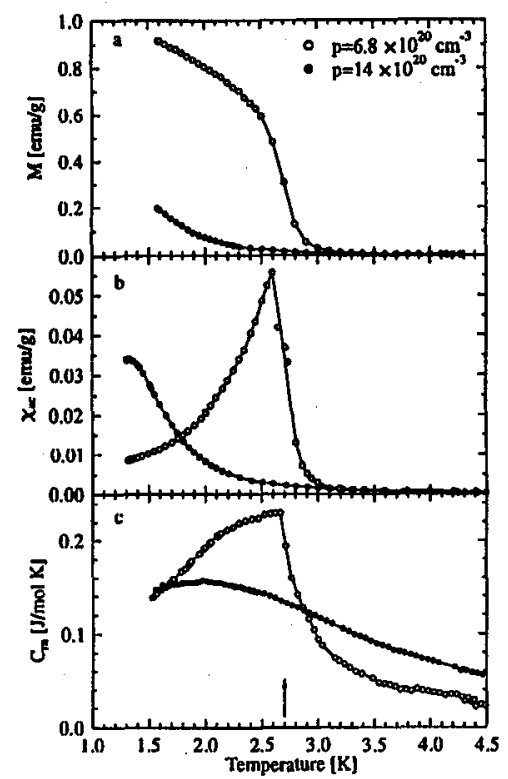

Fig. 2. The temperature dependence of magnetization (a), ac magnetic susceptibility (b) and magnetic contribution to the specific heat (c) of $\mathrm{Pb}_{0.26} \mathrm{Sn}_{0.72} \mathrm{Mn}_{0.02} \mathrm{Te}$ with two different carrier concentrations. The sample with $p=6.8 \times 10^{20} \mathrm{~cm}^{-3}$ shows a typical ferromagnetic behaviour. The ferromagnetic order is not observed in the sample with a higher carrier concentration. The arrow marks the paramagnetic Curie temperature for the sample with $p=14 \times 10^{20} \mathrm{~cm}^{-3}$.

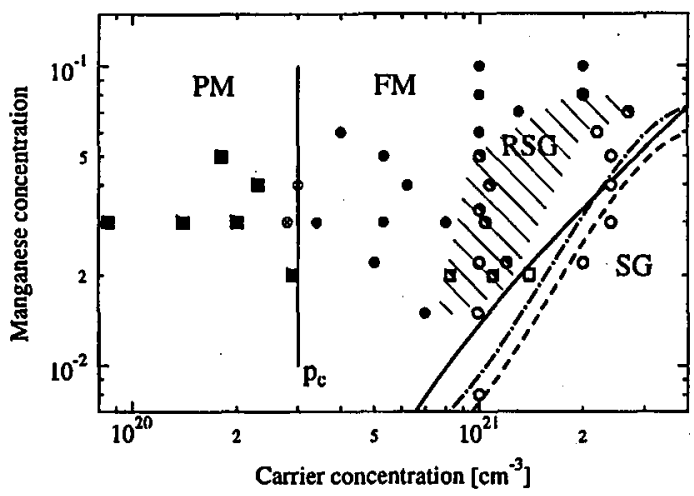

Fig. 3. $(x-p)$ magnetic phase diagram of IV-VI semimagnetic semiconductors. The lines separating ferromagnetic (FM, full symbols) and spin-glass (SG, open symbols) region are calculated within the geometrical model (solid line), the Sherrington-Kirkpatrick model (dashed line) and the Sherrington-Southern model (dash-dotted line). The samples with $p<p_{\mathrm{c}}$ are paramagnetic (PM, crossed symbols). The samples with a reentrant-spin-glass behaviour (RSG, half-filled symbols and hatched region) are also presented. 
presented in Fig. 3. The line separating paramagnetic (PM) and ferromagnetic (FM) regions is given by the condition $p=p_{\mathrm{c}}$, where $p_{\mathrm{c}}$ is the threshold carrier concentration already discussed in the previous section. The line separating ferromagnetic and spin-glass (SG) regions is given by the condition $x / p=$ const, which follows from the properties of the RKKY interaction and from the simple geometrical arguments outlined below.

Due to the oscillatory character of the RKKY interaction one can specify a characteristic distance $R_{0} \propto p^{-1 / 3}$, which corresponds to the first switch of the RKKY interaction from ferromagnetic to antiferromagnetic. The magnetic interactions in the RKKY-governed diluted magnetic system are dominated by ferromagnetic interactions for $R \ll R_{0}$, where $R \propto x^{-1 / 3}$ is the mean interspin distance. In the other case, $R \gg R_{0}$, the oscillatory character of the RKKY interaction leads to the formation of a spin-glass phase. The condition $R=R_{0}$ corresponds to $x / p=$ const line in the $x-p$ magnetic phase diagram. The diagram presented in Fig. 3 was recently analyzed in more quantitative way based on the statistical analysis of the distribution of exchange interactions and the Sherrington-Kirkpatrick and Sherrington-Southern models [25].

\section{IV-VI semimagnetic semiconductors with Gd}

$\mathrm{Gd}$ is known to incorporate into the IV-VI matrices as $\mathrm{Gd}^{3+}$ ion possessing well-localized spin-only magnetic moment with $S=7 / 2$ due to $4 f^{7}$ electrons. As $\mathrm{Gd}^{3+}$ ions substitute $\mathrm{Pb}^{2+}$ or $\mathrm{Sn}^{2+}$ ions they are electrically active centers showing a donor character experimentally observed in, e.g., $\mathrm{Pb}_{1-x} \mathrm{Gd}_{x}$ Te. Due to the strong localization of $4 f$ orbitals of $\mathrm{Gd}$ there exists only a quite weak antiferromagnetic $f-f$ exchange interaction between Gd ions [26]. Also the experimental studies of the $s p-f$ exchange interaction between the free carriers and Gd $4 f$ electrons revealed only a very weak interaction [27].

Recent experimental studies of the magnetic properties of $\mathrm{Sn}_{1-x} \mathrm{Gd}_{x} \mathrm{Te}$ revealed a possibility to enhance the strength of the $\mathrm{Gd}-\mathrm{Gd}$ antiferromagnetic exchange interaction by almost an order of magnitude by changing the concentration of carriers [28]. The experimental results are presented in Fig. 4a, where the paramagnetic Curie temperature $\Theta$ normalized per Gd concentration $x$ is plotted versus carrier concentration for different Gd contents. The parameter $\theta / x$ is proportional to the sum of all interspin exchange interactions present in the system. Remarkably, the strong resonant enhancement of $f-f$ interactions is observed only in $\mathrm{Sn}_{1-x} \mathrm{Gd}_{x}$ Te samples with $x<0.05$ and with carrier concentration $p \simeq(3 \pm 0.5) \times 10^{20} \mathrm{~cm}^{-3}$. For the crystals with higher Gd content the observed effect is much weaker. The transport measurements revealed also that in the very same crystals in which the $f-f$ interaction is enhanced, the carrier mobility is by a factor of 5 higher as compared to other SnGdTe samples (see Fig. 4b). These experimental findings provide another striking example of the correlation between magnetic and electronic properties of IV-VI semimagnetic semiconductors as will be discussed below.

The magnetic and electronic properties of SnGdTe can be consistently explained in the framework of a recently developed model of Gd ion in SnTe matrix [28]. The model assumes that Gd $5 d$ electron states are located at the energy level 

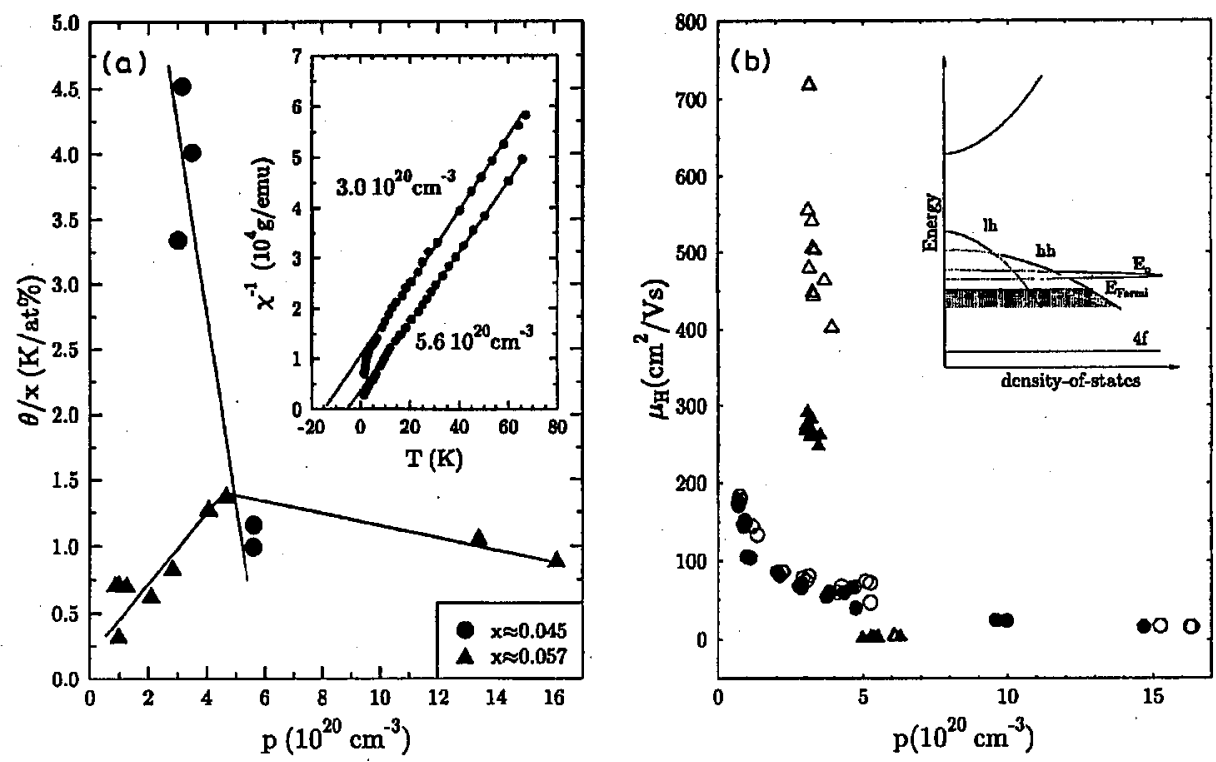

Fig. 4. (a) The carrier concentration dependence of the normalized paramagnetic Curie temperature $\Theta / x$ in $\operatorname{Sn}_{1-x} \mathrm{Gd}_{x}$ Te. Triangles - the behaviour of the crystals with $x>0.05$. Dots - the results for the samples with $x<0.05$. The inset presents the temperature dependence of the inverse magnetic susceptibility of two samples of $\mathrm{Sn}_{0.955} \mathrm{Gd}_{0.045} \mathrm{Te}$ with different carrier concentrations. (b) The carrier concentration dependence of the hole mobility for $\mathrm{Sn}_{1-x} \mathrm{Gd}_{x} \mathrm{Te}$ with $x=0.045$ (triangles) and with $x=0.057$ (circles). The open symbols show the data at $T=4.2 \mathrm{~K}$ whereas the full symbols correspond to $T=77 \mathrm{~K}$. The inset presents the scheme of the band structure of SnGdTe with $x<0.05$. With the increasing Gd content the $E_{0}$ electron level shifts up with respect to the top of the valence band leaving the band of heavy holes for $x \simeq 0.05$.

$E_{0}\left(\mathrm{Gd}^{2+/ 3+}\right.$ level) about $0.2 \mathrm{eV}$ below the top of the valence band (see the inset in Fig. $4 \mathrm{~b}$ ). The density of states related to $4 f^{7}$ energy states of Gd was experimentally found $9.5 \mathrm{eV}$ below the top of the valence band. It generates a new situation with $\mathrm{Gd}$ being in $3+$ or in $2+$ charge state depending on the relative position of the Fermi level (governed by the concentration of two electrically active centers: $\mathrm{Sn}$ vacancies and $\mathrm{Gd}^{3+}$ ions) and the $E_{0}$ energy level. In our model the $\mathrm{Gd} 5 d$ orbital may be empty (corresponding to the usual case of $4 f^{7} 5 d^{0}, \mathrm{Gd}^{3+}$ configuration) or occupied by just one electron (the case of $\mathrm{Gd}^{2+}$. with $4 f^{7} 5 d^{1}$ configuration).

The resonant enhancement of the Gd-Gd exchange interaction is explained within the framework of the two-stage $f-d-d-f$ exchange interaction mechanism. It consists of two steps: the intra-ion $4 f-5 d$ exchange interaction and the inter-ion $5 d-5 d$ coupling which may proceed both via free carriers or via anions. The first step is well known from the free ion data. The second step is also expected to be effective because of the considerably less localized character of the $5 d$ orbitals as compared to the $4 f$ orbitals. For this mechanism to be fully effective in SnGdTe 
one requires the following: the Fermi level must be located resonantly close to the $E_{0}$ level being, simultaneously, in the $\Sigma$-band of heavy holes with a high density of states. The analysis of the $\mathrm{Gd}$ composition dependence of the band structure of $\mathrm{SnGdTe}$ indicates that both these conditions can be fulfilled for $\mathrm{Sn}_{1-x} \mathrm{Gd}_{x} \mathrm{Te}$ with $x<0.05$. In these crystals the effect of the resonant enhancement of the $\mathrm{Gd}-\mathrm{Gd}$ exchange orientations is indeed observed. For the samples with $x>0.05$, the $E_{0}$ level of Gd is located above the top of the $\Sigma$-band (see Fig. $4 \mathrm{~b}$ ) and the resonant condition $E_{\mathrm{F}} \simeq E_{0}$ can be fulfilled only for light holes with a much smaller magnitude of the effect due to the low density of states at the Fermi level. The superposition of the resonant contribution due to the light holes and of the non-resonant contribution due to the heavy holes results in a non-monotonic carrier concentration dependence of the paramagnetic Curie temperature observed experimentally in $\mathrm{Sn}_{1-x} \mathrm{Gd}_{x} \mathrm{Te}$ with $x>0.05$.

The increase in the carrier mobility observed in the samples of $\mathrm{Sn}_{1-x} \mathrm{Gd}_{x} \mathrm{Te}$ with $x<0.05$ and $p=(3 \pm 0.5) \times 10^{20} \mathrm{~cm}^{-3}$ is also a consequence of the $E_{\mathrm{F}} \simeq E_{0}$ resonance. This situation corresponds to the case of the most of $\mathrm{Gd}$ ions being in the neutral 2+ charge state. As such, Gd ions are expected to be quite inefficient scattering centers. In the usual case of Fermi level located well below the $E_{0}$ level, all Gd ions are in electrically active $3+$ charge state constituting the main scattering centers and limiting the low temperature mobility of SnGdTe.

\section{Conclusions}

IV-VI semimagnetic semiconductors with $\mathrm{Mn}$ or with $\mathrm{Gd}$ reveal a variety of magnetic behaviours including the RKKY ferromagnetic and the RKKY spin-glass phases as well as the Curie-Weiss paramagnetic state with antiferromagnetic $d-d$ or $f-f$ interactions. The magnetic properties of these crystals can be controlled by changing the concentration of carriers or by controlling the Fermi level position. It is the consequence of the dependence of the dominant interspin exchange interactions (RKKY interaction in PbSnMnTe and $f-d-d-f$ exchange interaction in $\mathrm{SnGdTe}$ ) on the electronic properties of these semiconductors.

\section{Acknowledgments}

I would like to acknowledge the continuous support of Prof. R.R. Gałąza and the fruitful cooperation with Prof. W.J.M. de Jonge group at Eindhoven University of Technology. This work was supported in part by the Committee for Scientific Research (Poland) under project 2 P03B 10308.

\section{References}

[1] R.R. Gałązka, J. Magn. Magn. Mater. 140-144, 13 (1995).

[2] W.J.M. de Jonge, H.J.M. Swagten, J. Magn. Magn. Mater. 100, 322 (1991).

[3] Diluted Magnetic Semiconductors, Eds. J.K. Furdyna, J. Kossut, in series Semiconductors and Semimetals, Vol. 25, Academic Press, Boston 1988.

[4] J. Kossut, W. Dobrowolski, in: Handbook of Magnetic Materials, Ed. K.H.J. Buschow, Vol. 7, North-Holland, Amsterdam 1993, p. 231.

[5] M. Górska, J.R. Anderson, Phys. Rev. B 38, 9120 (1988). 
[6] G. Bauer, H. Pascher, W. Zawadzki, Semicond. Sci. Technol. 7, 703 (1992).

[7] T. Story, Z. Wilamowski, E. Grodzicka, W. Dobrowolski, B. Witkowska, J. Voiron, Acta Phys. Pol. A 87, 229 (1995).

[8] S. Isaber, C. Fau, S. Charar, M. Averous, Z. Golacki, Mater. Sci. Forum 182-184, 657 (1995).

[9] G. Nimtz, B. Schlicht, R. Dornhaus, Narrow Gap Semiconductors, Springer, Berlin 1983.

[10] T. Story, P.J.T. Eggenkamp. C.H.W. Swüste, H.J.M. Swagten, W.J.M. de Jonge, A. Szczerbakow, Phys. Rev. B 47, 227 (1993).

[11] G. Toth, J.Y. Leloup, H. Rodot, Phys. Rev. B 1, 4573 (1970).

[12] H. Lettenmayr, W. Jantsch, L. Palmetshofer, Solid State Commun. 64, 1253 (1987).

[13] B.A. Orłowski, B.J. Kowalski, R.R. Gałązka, N. Barret, K. Hrikovvini, M. Fang, C. Guillot, Solidi State Commun. 90, 139 (1994).

[14] T. Story, C.H.W. Swüste, P.J.T. Eggenkamp, H.J.M. Swagten, W.J.M. de Jonge, Phys. Rev. Lett. 77, 2802 (1996).

[15] T. Dietl, C. Śliwa, G. Bauer, H. Pascher, Phys. Rev. B 49, 2230 (1994).

[16] G. Karczewski, M. von Ortenberg, Z. Wilamowski, W. Dobrowolski, J. Niewodniczańska-Zawadzka, Solid State Commun. 55, 249 (1985).

[17] M. Escorne, A. Mauger, J.L. Tholence, R. Triboulet, Phys. Rev. B 29, 6306 (1986).

[18] T. Story, R.R. Gałązka, R.B. Frankel, P.A. Wolff, Phys. Rev. Lett. 56, 777 (1986).

[19] T. Story, G. Karczewski, L. Świerkowski, R.R. Gałązka, Phys. Rev. B 42, 10477 (1990).

[20] C.W.H.M. Vennix, E. Frikkee, P.J.T. Eggenkamp, H.J.M. Swagten, K. Kopinga, W.J.M. de Jonge, Phys. Rev. B 48, 3770 (1993).

[21] R.R. Gałązka, J. Spałek, A. Lewicki, B.C. Crooker, G. Karczewski, T. Story, Phys. Rev. B 43, 11093 (1991).

[22] H.J.M. Swagten, W.J.M. de Jonge, R.R. Gałązka, P. Warmenbol, J.T. Devreese, Phys. Rev. B 37, 9907 (1988).

[23] T. Story, P.J.T. Eggenkamp, C.H.W. Swüste, H.J.M. Swagten, W.J.M. de Jonge, L.F. Lemmens, Phys. Rev. B 45, 1660 (1992).

[24] W.J.M. de Jonge, T. Story, H.J.M. Swagten, P.J.T. Eggenkamp, Europhys. Lett. 17, 631 (1992).

[25] P.J.T. Eggenkamp, H.J.M. Swagten, T. Story, V.I. Litvinov, C.H.W. Swüste, W.J.M. de Jonge, Phys. Rev. B 51, 15250 (1995).

[26] M. Górska, J.R. Anderson, G. Kido, S.M. Green, Z. Gołacki, Phys. Rev. B 45, 11702 (1992).

[27] P. Urban, G. Sperlich, Solid State Commun. 16, 927 (1975).

[28] T. Story, M. Górska, A. Łusakowski, M. Arciszewska, W. Dobrowolski, E. Grodzicka, Z. Golacki, R.R. Gałazka, Phys. Rev. Lett. 77, 3447 (1996). 